\title{
Tissue induction, the relationship between biomaterial's microenvironment and mesenchymal stem cell differentiation
}

\author{
Wenfeng Xu ${ }^{1}$, Xiaoling Liao ${ }^{1}$, Ling Zhang ${ }^{1}$, Bo Liu ${ }^{2 *}$ \\ ${ }^{1}$ Biomaterials and Live Cell Imaging Institute, Chongqing University of Science and Technology, Chongqing, China \\ ${ }^{2}$ Department of Biomedical Engineering, Dalian University of Technology, Dalian, China \\ Email: ${ }^{*} 1$ bo@dlut.edu.cn
}

Received 22 October 2012; revised 23 November 2012; accepted 31 November 2012

\begin{abstract}
Mesenchymal stem cells (MSCs) have been recognized as the best candidate for tissue engineering, while the mechanism of biomaterial-induced MSCs differentiation is not well understood. Most of research has been focused on chemical signaling of biomaterial in the past, but a variety of non-chemical signals were also proved to play essential roles in cellular behaviors. In this paper, we reviewed the current reports about the effects of different kinds of biomaterial signals on MSCs differentiation.
\end{abstract}

Keywords: Biomaterial; Mesenchymal Stem Cells; Differentiation; Tissue Repair

\section{INTRODUCTION}

Cellular behaviors, including growth, survival, migration and differentiation, are regulated by the complex interplay between cells and their environment. For the rational design of functional artificial tissue or organ, it is vital to understand the mechanism of biomaterial-cell interaction and the possibility of manipulating these implants. While most papers focused on chemical signaling of biomaterial in the past, recent research emphasizes the effects of non-chemical signals, which showing the importance on cellular behaviors [1]. Increasing evidence proved that adhesion related non-chemical signals such as mechanical force and topography can play an equally important and complementary role [2-4]. Although there is a large volume of phenomenological records, the understanding of the mechanism of cellular responses to these signals remains fragmentary. In this paper, we describe the current reports about the important role of mesenchymal stem cells (MSCs) and biomaterials in tissue repair strategies, and also describe the common mechanism about the effect of material signal on the dif-

${ }^{*}$ Corresponding author. ferentiation of stem cells. This may help to develop new therapeutic interventions for devastating diseases such as cancer, and break through critical barriers facing the field of tissue engineering [5].

\section{TISSUE REPAIR MECHANISMS}

The main component involved in the tissue repair process includes the extracellular matrix and a variety of cells. Tissue repair process can hence be summarized with three well-coordinated steps: 1) Local inflammatory reaction: after the injury wound and fissure filled by blood clots, the trauma edge inflames and fibrin attaches at the meantime. Its purpose is to stop bleeding, close wounds and reduce the damage; 2) Cell regeneration and granulation tissue formation: the same kind of cells regenerate locally, and the granulation tissue is built with fibroblasts, endothelial cells and new blood vessels to fill the fractured organization, which will become the fibrous tissue (scar tissue) eventually as the frame to connect the damaged tissue. In addition to the fibroblasts, endothelial cells and epithelial cells, there are also chondrocytes, osteoblasts, mesenchymal cells and so on participate in this stage; 3) Tissue repair mould: damaged tissue can initially be repaired by cell proliferation and matrix deposition. However, such regenerated tissue does not meet the needs of physiological function entirely due to the formation of fibrous (scar) tissue and callus in quantity and quality.

With the recovery of body condition and activity, the nascent organization can be adjusted gradually. The speed and completeness of the tissue repair process can be affected by many factors, including the type of tissue damage, as well as the caused injury factor, nutrition, blood supply, infection and the scope of defected tissue. When the tissue is injured by the defect, the tissue structure and function can be restored completely under appropriate physiological conditions by regeneration from the damaged cells or the same kind of cell from adjacent 
tissue. This is called regenerative repair or completely repair, such as endometrium, gastrointestinal mucosa, and urinary tract mucous gland tissue repair. But the regenerative capacity is various among different cell types. Some of them have weak or even no regenerative capacity. These cells are out of cell cycle and in permanent cessation of mitosis, such as nerve cells, myocardial cells and skeletal muscle cells. So the manual intervention is needed in tissue regeneration for the goal of repair tissue perfectly.

\section{MESENCHYMAL STEM CELLS}

MSCs are the non-hematopoietic stem cells isolated from bone marrow, with high ability of proliferation, selfupdate and multi-differentiation potential. It's multipotential stem cell which can differentiate not only into mesodermal cells including osteoblasts, hematopoiesissupporting stromal cells, adipocytes, chondrocytes, myocytes and endothelial cells, but also nerve ectoderm tissue cell [6-9]. They play critical roles in the tissues establishment, regeneration and replacement due to their unique capability of both differentiation and self-renewal. So MSCs can be applied clinically as a new therapy for treating any tissue diseases with mesenchymal origin [10]. Numerous studies have reported beneficial effects of MSCs on tissue repair and regeneration in the clinical treatment of many diseases [11]. According to these researches, MSCs' characteristics can be summarized as following: 1) multilineage differentiation potential, including bone, cartilage, fat, muscle, nerve cells under different induction conditions in vitro; 2) easily available from a variety of tissue, such as bone marrow, umbilical cord blood, umbilical cord, placenta, adipose tissue, dermis, heart, liver, spleen, pulp, etc.; 3 ) easily expanded in vitro, and can still remain original features after several passages; 4) do not involve the ethical issues; 5) do not cause immune rejection. Because of these capabilities, MSCs have been recognized as the best candidate for tissue engineering, gene therapy and regenerative medicine for the treatment of many tissue diseases [12].

\section{BIOMATERIALS FOR TISSUE INDUCTION AND PROTEIN ADSORPTION}

The body tissue defects caused by trauma, tumor, inflammation or aging population have become one of the major clinical problems all over the world. Although fairly successful, current treatments including drugs delivery, surgical operation and tissue transplantation, do not provide optimum therapy to cure, because the drugs delivery will just delay the lesions and the surgery will cause tissue loss, and the latter raises the issue of supply and the risk of rejection or disease transfer [13]. In recent years, tissue engineering has won the increasing support as a therapy of tissue defects, which is a more effective approach than traditional methods. In particular, the tissue inducing of biomaterials is the basis of tissue engineering. In most cases controlled directly by biomaterial signals, tissue engineering attempts to stimulate stem cell differentiation to specific cell lines, and then organize into functional tissue assembly [14].

With the development of materials, biomedical and bionics science, some biomaterials have been widely used in tissue reparation. Up to now, the biomaterials have been developed from the first generation of "bioinert" material, the second generation of "bioactive" material, to the third generation of "tissue inducing" material. The "tissue inducing" materials should have excellent bioactivity, which induces cell attachment, growth and proliferation to achieve complete regeneration of tissues. Therefore, the biomaterial is no longer for tissue replacement, but rather for promote tissue regeneration [10]. These advances have led to clinical successes for inducing some simple tissues such as skin and cartilage [14].

For tissue inducing biomaterials, protein (e.g. growth factors) adsorption on the biomaterial surface and their impact on the MSCs behavior is the key for implants evaluation. Biomaterials might have good biocompatibility as a superior ability for selective protein adsorption, which depending on crystallite size, pore distribution and other structural factors [15]. A protein competitive adsorption process happens after the material implanted into the body, and material surface plays an important role in the protein selective adsorption, which affects cell selective adhesion and directed differentiation of MSCs [16-17]. Generally, the protein adsorption (e.g. growth factor) is rarely in the normal condition, but is significant in the growth of new tissue [18].

When the material been implanted in vivo, the interacttion happens firstly at the surface/interface between materials and the body, and then protein adsorption layer forms soon, which will affect subsequent cell behavior. After cells touch with the protein layer, the integrin receptors first interact with the protein molecules at contact location and transmit signaling to the cytoplasm, thus affect cell behavior and function. Generally, it is considered that the surface composition and structure of biomaterials affect cell behavior, in particular, the micro/nano surface structure lead to the protein conformational changes, thereby exposing the active binding domain to integrate with the cell. The surface structural characteristic determines the type of the adsorbed protein, and its conformation also further changes to specific cell adhesion, and then affects cell behavior. For example, Zhang's group showed that nano-biphasic calcium phosphate ceramic is able to selectively adsorb more low 
molecular weight of lysozyme (LSZ) in lysozyme/bovine serum albumin (BSA) protein system than conventional ceramics, which can significantly up-regulate the osteoblaste upstream gene of BMP2 in osteogenic cells in vitro, and induce more bone tissue in animal body [19].

\section{BIOMATERIAL ENVIRONMENTAL EFFECTS ON MESENCHYMAL STEM CELLS DIFFERENTIATION}

Biomaterials have played a crucial role in the development of tissue engineering. Current efforts are made to optimize the mechanical, physical, chemical and biological properties of biomaterials for applications which requiring different microenvironments [4]. One example is the design of surfaces that exhibit dynamic changes in interfacial properties, such as wettability, in response to an electrical potential [20]. Hence, a large number of exploratory studies on the induction mechanism of the biomaterial have been carried out, and generally they believed that biological materials play a key role in the regulation of stem cell differentiation behavior in tissue induction, which are closely related to the biomaterial's composition and structure, especially surface chemistry, physical, biomechanical characteristics and geometric characteristics [21-23].

\subsection{The Effect of Surface Chemistry Properties}

Material-driven control of bone-marrow-derived MSCs behavior and differentiation is very exciting in biomaterials field. Many researching results have found that material surface signal such as surface chemistry can regular MSCs behaviour, including initial protein and cell adhesion/morphology and ultimately differentiation. Judith M. Curran modified the glass silane surface with methyl $\left(-\mathrm{CH}_{3}\right)$, amino $\left(-\mathrm{NH}_{2}\right)$, silane $(-\mathrm{SH})$, hydroxyl $(-\mathrm{OH})$ and carboxyl $(-\mathrm{COOH})$ respectively, and evaluate the defined surface chemistry on MSC behavior [23]. The results demonstrate- $\mathrm{CH}_{3}$ surfaces maintained the MSC phenoltype, the $-\mathrm{NH}_{2}$ and - $\mathrm{SH}$ modified surfaces promoted osteogenesis, $-\mathrm{OH}$ and $-\mathrm{COOH}$ modified surfaces promoted chondrogenesis. Benjamin G. Keselowsky investigated the inferences of surface chemistry with well-defined chemistries $\left(\mathrm{CH}_{3}, \mathrm{OH}, \mathrm{COOH}, \mathrm{NH}_{2}\right)$ on focal adhesion assembly and signaling protein [22]. The results showed that surface chemistry functional groups strongly modulated integrin binding, such as integrin $\alpha 5 \beta 1$ binding affinity followed the order $\mathrm{OH}>\mathrm{NH}_{2}=\mathrm{COOH}>\mathrm{CH}_{3}$, while integrin $\alpha_{\mathrm{V}} \beta_{3}$ displayed the connection $\mathrm{COOH}>$ $\mathrm{NH}_{2} \geqq \mathrm{OH}=\mathrm{CH}_{3}$; Their binding affinity affect the adhesion structure and the types of protein molecules, and the $\mathrm{OH}$ supported the highest expression levels of talin, $\alpha$-actin, paxillin, and tyrosine phosphorylated proteins in the adhesive structures, while the $\mathrm{NH}_{2}$ and
$\mathrm{COOH}$ surfaces exhibited intermediate level and $\mathrm{CH}_{3}$ substrate displayed the lowest. It implies that the pattern in focal adhesion assembly correlated well with integrin $\alpha \beta$ subunit binding. Other research has shown that the $\mathrm{NH}_{2}$ and $\mathrm{SH}$ groups can promote osteoblast differentiation, and $\mathrm{OH}$ and $\mathrm{COOH}$ groups can induce cartilage cells generation [23].

\subsection{The Effect of Surface Physical Properties}

The surface physical properties of biomaterials, including the spatial structure and topography at a single cell scale may regulate cells spreading shape and thickness, retaining contact between cells. Some hierarchical cluster analysis showed that scaffold structure was more influential than scaffold composition. It has been evaluated that the quasi 3-d structure may be more close to nature environment than 2-D smooth substrate [24], which showed improved cell spreading and proliferation on topographical substrate and inhibited MSCs osteogenicdifferentiation on grooved substrate. It is known that surface topography from the micro- to the nanoscale can direct cell function. Especially, the effects of surface topography on cell behavior were mediated by cell shape [24]. For example, when using the fibronectin (FN) with square micropatterns, the size of the micro-pattern can induce MSCs to differentiate into different cell through changing the stem cells shape. MSCs adhesion on the micropatterns with $10,000 \mu^{2}$ differentiated into osteoblasts, while on $1024 \mu \mathrm{m}^{2}$ differentiated into adipocytes [25]. Kilian found that geometric features that increase actomyosin contractility promote osteogenesis and are consistent with characteristics of the microenvironment in vivo of the differentiated cells [26]. Guvendiren reported that human MSCs attached to lamellar wrinkles spread by taking the shape of the pattern with strong directionality, and differentiate into an osteogenic lineage, while cells that attached inside the hexagonal patterns remain rounded with low spreading and differentiate into an adipogenic lineage [27].

The micro-structural features of biomaterial surface can also affect MSCs differentiation, but its mechanism has not been clearly clarified yet. Previous studies found that the different micro-patterned ECM surface of biomaterial has a significant effect on the adsorption quantity of protein and life behavior of stem cells [25]. These results showed that micro-patterned size not only controls the adsorption quantity of bone growth factor protein, but also impacts on cell adhesion and morphology, as well as protein expression during the cell growth process [28]. Compared with un-micro-patterned plane biomaterials, micro-patterned surface can promote cells to secrete more type II collagen and type VI collagen, which indicates its role in regulation of cell growth [27]; On the other hand, even with the same size and shape of micro- 
pattern, different ECM protein micro-patterns have significant different effects on cell adhesion and protein expression. It implies that the morphology of micropatterned bioactive molecules can not only selectively control the protein adsorption, but also achieve the selective growth of MSCs, and then affect its differentiation and enhance the biochemical binding capacity of biomaterial with the cell.

\subsection{The Effect of Mechanical Properties}

The cell is also very sensitive to the traction caused by surface morphology and hardness of biomaterials, which confirming that even a small stress can also affect the differentiation of stem cell [29-31]. It was reported that stem cells commit to the directly differentiation lineage specified by matrix elasticity, which was differentiated into nerve cells with the elastic modulus from 0.1 to 1 $\mathrm{KPa}$, muscle cell with 8 - $17 \mathrm{KPa}$ interval, and osteoblast with $25-40 \mathrm{KPa}$ [1]. Histological studies have shown that hard hydroxyapatite can induce bone formation [32], while soft collagen-based hydrogels can induce cartilage formation [33]. Shu Chien's team recently revealed that the hardness-induced MSCs differentiation may be associated with integrin activation and internalization at the adhesion sites within the phenomenon of cell experiments [34]. Park et al. found that soft substrate inhibit MSCs spreading through suppression of Rho to reduce stress fibers and $\alpha$-actin synthesis, but the cartilage and fat cell markers collagen-II and lipoprotein lipase (LPL) were highly expressed [35].

In conclusion, the multilineage differentiation potential of MSCs is controlled by their interactions with the microenvironment or niche consisting of biomaterials science factors (such as macromolecules, rigidity, topography and hydrophobicity, etc.), chemical stimuli (growth factor, etc.), associated with mechanical stimuli (flow, pressure, stretch, etc.). Simulating the natural ECM with intricate and highly ordered structure is still a challenging work. Thus, for the purpose of reconstituting an optimal microenvironment for MSCs in vitro, many researches focused on controlling the biomaterials science factors in tissue engineering, and the establishment of unique tissue-specific ECM will facilitate the control of MSCs fate for therapeutic applications [36].

\section{SUMMARY AND PERSPECTIVES}

Obviously, the results mentioned above suggest that the behavior of materials science signal intrinsically linked to the differentiation of stem cells. However, most research of its mechanism is currently limited to a single factor of the material on stem cell behavior and gene expression, or based on randomly selected substrate surface, and there is a lack of comprehensive evaluation on multi-factor materials science, even though the biomaterial often includes a variety of concentrated materials science signal. It is difficult to precisely define the morphology of biomaterial, and these studies tend to be more understanding of the phenomenon than mechanism. Hence, sometime the effect of surface morphology and topology on protein adsorption and MSCs growth behavior are overlooked. Therefore, the regulated MSCs growth has not been achieved on some kind of material such as the carbon/carbon surface, and there was still no design of a specific recognizable surface by cell, and thus the truly healing cannot be achieved between the implant material and human tissue. At the same time, the material organization must be induced through the expression and activity regulation of the corresponding protein molecules in living cells. Due to the lack of evaluation system of biomaterial organization induced from living cell at the molecular level, its mechanism remains unclear.

The combinatorial approach of tissue engineering to biomaterials and cell heterozygote provide a method to select the optimum biomaterials for controlling stem cell fate. Recent advances in biomaterials have led to the identification of polymer substrates for self-renewal of stem cell [10]. These biomaterials have the advantage of being cost-effective and being non-biological, in addition to the scalable ability. However, undefined components caused obscure results of basic research (e.g. role of signaling molecules in stem cell self-renewal), and the microenvironmental conditions used may not be optimal. For that purpose, some researchers have applied the high-throughput technology to select polymers from a library that can support self-renewal of stem cell with the maintenance of pluripotency [37-39]. Some other research formed microvessel-like structures to establish a high-throughput screening platform for identification of macromolecules and their interactions with growth factors to optimize the microenviromental factors to modulate stem cell fate [40]. These explorations helped the understanding of biomaterial-induced MSCs differentiation and also promoted the design of new biomaterial.

How materials science signals regulate directed differentiation of stem cell to specific tissue cell lines, thus induce repair and regeneration of tissues and organs? These questions are lack of understanding from molecular level of living cell, and the mechanism of tissue induction has not been elucidated. Fluorescence Resonance Energy Transfer (FRET) technology not only determines the size of the protein activity changes, but also can be directly used to observe the change of spatial distribution of protein activation in living cell [41]. A lot of experiments have proved that FRET technology is very reliable and effective in the observation and quantitative analysis of the spatiotemporal changes of live intracellular signal 
transmission $[42,43]$. Since its inception, FRET has been widely used in exploring the pathogenesis of various diseases such as cancer, neurological and cardiovascular disease. However, the application of FRET in tissue engineering to explore the mechanisms of materials science signal directed stem cell differentiation at the molecular level still has been rarely reported. Our group's early study has shown that, the real-time observation and quantitative analysis of the effect of extracellular matrix (such as the stimulation of fibronectin and collagen) on the adhesion and spreading shape of living MSCs, the change of FAK signal protein activity and distribution, can be achieved by using FRET technology. Our studies have also confirmed that a quantitative relationship exists between the biomaterials science signal and FAK signaling activity [41]. These explorations indicated that FRET could be widely used in biomaterials science field to reveal the mechanism of material-induced MSCs differentiation.

In addition, most of the in vitro models about regenerative medicine are performed to confirm cell phenoltype under the simulated physiological conditions. However, a regenerative process is not based on a quiescent physiological niche. Cells constantly probe and respond to a myriad of dynamic cues present in their local surroundings [44], and the neighboring cells will affect its behavior directly or indirectly. In vitro systems using co-culture can be designed to simulate the in vivo situation (human origin, 3D-systems, etc.) more closely. Cocultures of human cells on biomaterials can provide better understanding of tissue engineering principles, such as how cells interact with microstructure, especially uptake and transport of nanoparticles, which dependent on physicochemistry, cell type, mode of administration, etc.

If a high-throughput screening system with biological material array of different chemical stimulation, roughness, hydrophilic, hardness, or even 3D structure and co-culture method, could be combined with FRET technology to analysis the spatiotemporal signaling protein activity and distribution within MSCs under the stimulus of different materials science, it will be expected not only to establish a new set of assessment system in biomaterial tissue inductivity, but also provides a more direct thought and method for optimal design of biomaterials in the future.

\section{ACKNOWLEDGEMENTS}

This work was supported in part by grants from National Natural Science Foundation of China (NSFC) 10972139, 31271014, and Natural Science Key Foundation Project of CQ in China (CSTC2012JJB0097).

\section{REFERENCES}

[1] Engler, A.J., Sen, S., Sweeney, H.L. and Discher, D.E.
(2006) Matrix elasticity directs stem cell lineage specification. Cell, 126, 677-689. doi:10.1016/j.cell.2006.06.044

[2] Graziano, A., d'Aquino, R., Cusella-De Angelis, M.G., De Francesco, F., Giordano, A., Laino, G., Piattelli, A., Traini, T., De Rosa, A. and Papaccio, G. (2008) Scaffold's surface geometry significantly affects human stem cell bone tissue engineering. Journal of Cellular Physiology, 214, 166-172. doi:10.1002/jcp.21175

[3] Seo, C.H., Furukawa, K., Montagne, K., Jeong, H. and Ushida, T. (2011) The effect of substrate microtopography on focal adhesion maturation and actin organization via the RhoA/ROCK pathway. Biomaterials, 32, 95689575. doi:10.1016/j.biomaterials.2011.08.077

[4] Zhang, H., Dai, S., Bi, J. and Liu, K. (2011) Biomimetic three-dimensional microenvironment for controlling stem cell fate. Interface Focus, 1, 792-803. doi:10.1098/rsfs.2011.0035

[5] Wagoner, J.A. and Harley, B.A.C. (2011) Mechanobiology of cell-cell and cell-matrix interactions. Springer, Berlin. doi:10.1007/978-1-4419-8083-0

[6] Oh, S., Brammer, K.S., Li, Y.S., Teng, D., Engler, A.J., Chien, S. and Jin, S. (2009) Stem cell fate dictated solely by altered nanotube dimension. Proceedings of the $\mathrm{Na}$ tional Academy of Sciences of the USA, 106, 2130-2135. doi:10.1073/pnas.0813200106

[7] Prockop, D.J. (1997) Marrow stromal cells as stem cells for nonhematopoietic tissues. Science, 276, 71-74. doi:10.1126/science.276.5309.71

[8] Dennis, J.E., Merriam, A., Awadallah, A., Yoo, J.U., Johnstone, B. and Caplan, A.I. (1999) A quadripotential mesenchymal progenitor cell isolated from the marrow of an adult mouse. Journal of Bone and Mineral Research, 14, 700-709. doi:10.1359/jbmr.1999.14.5.700

[9] Ferrari, G., Cusella-De Angelis, G., Coletta, M., Paolucci, E., Stornaiuolo, A., Cossu, G. and Mavilio, F. (1998) Muscle regeneration by bone marrow-derived myogenic progenitors. Science, 279, 1528-1530. doi:10.1126/science.279.5356.1528

[10] Liao, X., Lu, S., Zhuo, Y., Winter, C., Xu, W., Li, B. and Wang, Y. (2011) Bone physiology, biomaterial and the effect of mechanical/physical microenvironment on mesenchymal stem cell osteogenesis. Cellular and Molecular Bioengineering, 4, 579-590. doi:10.1007/s12195-011-0204-9

[11] Zandstra, P.W. and Nagy, A. (2001) Stem cell bioengineering. Annual Review of Biomedical Engineering, 3, 275-305. doi:10.1146/annurev.bioeng.3.1.275

[12] Sun, Y., Li, W., Lu, Z., Chen, R., Ling, J., Ran, Q., Jilka, R.L. and Chen, X.D. (2011) Rescuing replication and osteogenesis of aged mesenchymal stem cells by exposure to a young extracellular matrix. FASEB Journal, 25, 1474-1485. doi:10.1096/fj.10-161497

[13] Laurencin, C.T., Ambrosio, A.M., Borden, M.D. and Cooper Jr., J.A. (1999) Tissue engineering: Orthopedic applications. Annual Review of Biomedical Engineering, 1, 19-46. doi:10.1146/annurev.bioeng.1.1.19

[14] Langer, R. (2007) Tissue engineering: Perspectives, challenges, and future directions. Tissue Engineering, 13, 1-2. 
doi:10.1089/ten.2006.0219

[15] Fujii, E., Ohkubo, M., Tsuru, K., Hayakawa, S., Osaka, A., Kawabata, K., Bonhomme, C. and Babonneau, F. (2006) Selective protein adsorption property and characterization of nano-crystalline zinc-containing hydroxyapatite. Acta Biomaterialia, 2, 69-74. doi:10.1016/j.actbio.2005.09.002

[16] Huebsch, N. and Mooney, D.J. (2009) Inspiration and application in the evolution of biomaterials. Nature, 462, 426-432. doi:10.1038/nature08601

[17] Scopelliti, P.E., Borgonovo, A., Indrieri, M., Giorgetti, L., Bongiorno, G., Carbone, R., Podesta, A. and Milani, P. (2010) The effect of surface nanometre-scale morphology on protein adsorption. PLoS One, 5, e11862. doi:10.1371/journal.pone.0011862

[18] Eguchi, Y., Wakitani, S., Naka, Y., Nakamura, H. and Takaoka, K. (2011) An injectable composite material containing bone morphogenetic protein-2 shortens the period of distraction osteogenesis in vivo. Journal of Orthopaedic Research, 29, 452-456. doi:10.1002/jor.21225

[19] Hong, Y., Fan, H., Li, B., Guo, B., Liu, M. and Zhang, X. (2010) Fabrication, biological effects, and medical applications of calcium phosphate nanoceramics. Materials Science and Engineering $R$, 70, 225-242. doi:10.1016/j.mser.2010.06.010

[20] Lahann, J., Mitragotri, S., Tran, T.N., Kaido, H., Sundaram, J., Choi, I.S., Hoffer, S., Somorjai, G.A. and Langer, R. (2003) A reversibly switching surface. Science, 299, 371-374. doi:10.1126/science.1078933

[21] Martino, S., D’Angelo, F., Armentano, I., Kenny, J.M. and Orlacchio, A. (2012) Stem cell-biomaterial interactions for regenerative medicine. Biotechnology Advances, 30, 338-351. doi:10.1016/j.biotechadv.2011.06.015

[22] Keselowsky, B.G., Collard, D.M. and Garcia, A.J. (2004) Surface chemistry modulates focal adhesion composition and signaling through changes in integrin binding. Biomaterials, 25, 5947-5954.

doi:10.1016/j.biomaterials.2004.01.062

[23] Curran, J.M., Chen, R. and Hunt, J.A. (2006) The guidance of human mesenchymal stem cell differentiation in vitro by controlled modifications to the cell substrate. Biomaterials, 27, 4783-4793. doi:10.1016/j.biomaterials.2006.05.001

[24] Kumar, G., Tison, C.K., Chatterjee, K., Pine, P.S., McDaniel, J.H., Salit, M.L., Young, M.F. and Simon Jr., C.G. (2011) The determination of stem cell fate by 3D scaffold structures through the control of cell shape. Biomaterials, 32, 9188-9196. doi:10.1016/j.biomaterials.2011.08.054

[25] McBeath, R., Pirone, D.M., Nelson, C.M., Bhadriraju, K. and Chen, C.S. (2004) Cell shape, cytoskeletal tension, and RhoA regulate stem cell lineage commitment. Developmental Cell, 6, 483-495.

[26] Kilian, K.A., Bugarija, B., Lahn, B.T. and Mrksich, M. (2010) Geometric cues for directing the differentiation of mesenchymal stem cells. Proceedings of the National Academy of Sciences of the USA, 107, 4872-4877. doi:10.1073/pnas.0903269107
[27] Guvendiren, M. and Burdick, J.A. (2010) The control of stem cell morphology and differentiation by hydrogel surface wrinkles. Biomaterials, 31, 6511-6518. doi:10.1016/j.biomaterials.2010.05.037

[28] Namgung, S., Baik, K.Y., Park, J. and Hong, S. (2011) Controlling the growth and differentiation of human mesenchymal stem cells by the arrangement of individual carbon nanotubes. ACS Nano, 5, 7383-7390. doi:10.1021/nn2023057

[29] Anselme, K., Ponche, A. and Bigerelle, M. (2010) Relative influence of surface topography and surface chemistry on cell response to bone implant materials. Part 2: Biological aspects. Proceedings of the Institution of Mechanical Engineers, Part H, 224, 1487-1507.

[30] Kong, H.J., Polte, T.R., Alsberg, E. and Mooney, D.J. (2005) FRET measurements of cell-traction forces and nano-scale clustering of adhesion ligands varied by substrate stiffness. Proceedings of the National Academy of Sciences of the USA, 102, 4300-4305. doi:10.1073/pnas.0405873102

[31] Chowdhury, F., Na, S., Li, D., Poh, Y.C., Tanaka, T.S., Wang, F. and Wang, N. (2010) Material properties of the cell dictate stress-induced spreading and differentiation in embryonic stem cells. Nature Materials, 9, 82-88. doi: $10.1038 /$ nmat 2563

[32] Zhang, X., Zou, P., Wu, C., Qu, Y. and Jg, Z. (1991) A study of porous blocks HA ceramics and its osteogeneses. Elsevier, New York, 408-415.

[33] Zhang, L., Li, K.F., Xiao, W.Q., Zheng, L., Xiao, Y.M., Fan, H.S. and Zhang, X.D. (2011) Preparation of collagen-chondroitin sulfate-hyaluronic acid hybrid hydrogel scaffolds and cell compatibility in vitro. Carbohydrate Polymers, 84, 118-125. doi:10.1016/j.carbpol.2010.11.009

[34] Du, J., Chen, X., Liang, X., Zhang, G., Xu, J., He, L., Zhan, Q., Feng, X.Q., Chien, S. and Yang, C. (2011) Integrin activation and internalization on soft ECM as a mechanism of induction of stem cell differentiation by ECM elasticity. Proceedings of the National Academy of Sciences of the USA, 108, 9466-9471. doi:10.1073/pnas.1106467108

[35] Park, J.S., Chu, J.S., Tsou, A.D., Diop, R., Tang, Z., Wang, A. and Li, S. (2011) The effect of matrix stiffness on the differentiation of mesenchymal stem cells in response to TGF-beta. Biomaterials, 32, 3921-3930. doi:10.1016/j.biomaterials.2011.02.019

[36] Lai, Y., Sun, Y., Skinner, C.M., Son, E.L., Lu, Z., Tuan, R.S., Jilka, R.L., Ling, J. and Chen, X.D. (2010) Reconstitution of marrow-derived extracellular matrix ex vivo: A robust culture system for expanding large-scale highly functional human mesenchymal stem cells. Stem Cells and Development, 19, 1095-1107. doi: $10.1089 / \mathrm{scd} .2009 .0217$

[37] Wu, S.M., Cybart, S.A., Yu, P., Rossell, M.D., Zhang, J.X., Ramesh, R. and Dynes, R.C. (2010) Reversible electric control of exchange bias in a multiferroic fieldeffect device. Nature Materials, 9, 756-761. doi: $10.1038 /$ nmat 2803

[38] Rodin, S., Domogatskaya, A., Strom, S., Hansson, E.M., 
Chien, K.R., Inzunza, J., Hovatta, O. and Tryggvason, K. (2010) Long-term self-renewal of human pluripotent stem cells on human recombinant laminin-511. Nature Biotechnology, 28, 611-615. doi:10.1038/nbt.1620

[39] Villa-Diaz, L.G., Nandivada, H., Ding, J., Nogueira-deSouza, N.C., Krebsbach, P.H., O’Shea, K.S., Lahann, J. and Smith, G.D. (2010) Synthetic polymer coatings for long-term growth of human embryonic stem cells. Nature Biotechnology, 28, 581-583. doi:10.1038/nbt.1631

[40] Kolbe, M., Xiang, Z., Dohle, E., Tonak, M., Kirkpatrick, C.J. and Fuchs, S. (2011) Paracrine effects influenced by cell culture medium and consequences on microvessellike structures in cocultures of mesenchymal stem cells and outgrowth endothelial cells. Tissue Engineering Part $A, 17,2199-2212$. doi:10.1089/ten.tea.2010.0474

[41] Liao, X., Lu, S., Zhuo, Y., Winter, C., Xu, W. and Wang,
Y. (2012) Visualization of Src and FAK Activity during the Differentiation Process from HMSCs to Osteoblasts. PLoS One, 7, e42709. doi:10.1371/journal.pone.0042709

[42] Liu, B, Kim, T.J. and Wang, Y. (2010) Live cell imaging of mechanotransduction. Journal of the Royal Society Interface, 7, S365-S375. doi:10.1098/rsif.2010.0042.focus

[43] Wang, Y., Shyy, J.Y. and Chien, S. (2008) Fluorescence proteins, live-cell imaging, and mechanobiology: Seeing is believing. Annual Review of Biomedical Engineering, 10, 1-38. doi:10.1146/annurev.bioeng.010308.161731

[44] Choi, C.K., Breckenridge, M.T. and Chen, C.S. (2010) Engineered materials and the cellular microenvironment: A strengthening interface between cell biology and bioengineering. Trends in Cell Biology, 20, 705-714. doi:10.1016/j.tcb.2010.09.007 